\title{
The autotransporter secretion system
}

\author{
Mickaël Desvaux, Nicholas J. Parham, Ian R. Henderson * \\ Bacterial Pathogenesis and Genomics Unit, Division of Immunity and Infection, The Medical School, University of Birmingham, Edgbaston, \\ Birmingham, B15 2TT, UK
}

Received 9 September 2003; accepted 3 October 2003

First published online 7 October 2003

\begin{abstract}
The type V secretion system includes the autotransporter family, the two-partner system and the Oca family. The autotransporter secretion process involving first the translocation of the precursor through the inner membrane and then its translocation through the outer membrane via a pore formed by a $\beta$-barrel is reviewed.
\end{abstract}

(C) 2003 Elsevier SAS. All rights reserved.

Keywords: Autotransporter; Membrane transport protein; Protein translocation; Virulence factors

\section{Introduction}

In contrast to Gram-positive bacteria which possess only one biological membrane, called the cytoplasmic membrane, the cell envelope of Gram-negative bacteria is composed of two biological membranes, the inner membrane, i.e., the cytoplasmic membrane, and the outer membrane. This dual membrane envelope presents a real challenge for the transport of molecules into and out of these microorganisms. The diversity of the proteinaceous transport systems currently recognized in Gram-negative bacteria far exceeds that recognized in the Gram-positive bacteria, Archaea and Eukarya [62]. To date, most of the information concerning the bacterial protein secretion mechanisms is derived from studies of pathogenic bacteria. In fact, the capacity of a bacterium to infect, multiply and survive in contact with or inside a host cell depends greatly on its capacity to secrete proteinaceous virulence factors such as adhesin, toxins, enzymes and/or mediators of motility.

The existence of protein secretion per se first implies the existence of systems allowing the transport through the cell envelope, i.e., from the inside to the outside of the cell, and second that the secreted proteins possess defined information targeting them toward the cytoplasmic membrane. In Grampositive bacteria the translocation through the cytoplasmic

\footnotetext{
* Corresponding author.

E-mail address: I.R.Henderson@bham.ac.uk (I.R. Henderson).
}

membrane corresponds to secretion while in Gram-negative bacteria it corresponds to export into the periplasm, thus to arrive in the extracellular environment proteins must still cross the outer membrane barrier. Hence, in contrast to other members of the Procarya, protein secretion in Gram-negative bacteria is governed by the translocation of the secreted protein through the outer membrane and not through the cytoplasmic membrane.

In Gram-negative bacteria, two inner membrane translocation systems involved in protein secretion have been identified. These protein complexes called translocases are composed of (i) transmembrane structural proteins forming the translocon, and (ii) some accessory factors. The most recently discovered system is the twin-arginine translocation (Tat) pathway composed of the TatABCE translocon [4], while the Sec (secretion) system involves the SecYEG translocon, a protein conducting channel, and the cytoplasmic ATPase, SecA, which provides the energy for translocation $[18,33,53]$. Two distinct protein targeting pathways converge at the Sec translocon: the SRP (signal recognition particle)-dependent and the SecB-dependent pathways [77].

The molecular mechanism involved in the targeting and the transport of proteins through the Tat translocon is not well understood. It is hypothesised that the secreted protein binds directly to Tat translocon components before a $\Delta \mathrm{p}$-driven translocation occurs. An alternative model has recently been proposed whereby membrane integration could precede Tat-dependent translocation and the membrane targeting process may require energy from ATP-dependent 
$\mathrm{N}$-terminal unfolding steps [5]. The major feature of the Tat pathway is that it is capable of transporting proteins in a native state [60]. In contrast to the Tat-system, the Secdependent pathway translocates proteins in an extended conformation where SecB permits a posttranslational translocation and SRP allows a co-translational translocation of the protein $[43,76]$. However, it has been demonstrated that some proteins are routed to the Sec system via SecB- and SRP-independent pathways [2,58].

To date, five secretion pathways permitting the secretion of soluble proteins outside the cellular compartment have been described [28,63]. It is worth remembering that this classification, where the secretion pathways have been numbered from I to $\mathrm{V}$, is restricted to the Gram-negative bacteria only. After a brief summary of the other secretion pathways, this review will focus on the type $\mathrm{V}$ secretion system and more particularly on autotransporters with a special interest in the molecular mechanisms involved in the course of secretion.

\section{Protein secretion in Gram-negative bacteria}

The secretion pathway classification in Gram-negative bacteria is categorized primarily by the outer membrane translocation mechanisms.

The type I secretion system (TOSS) is exemplified by the HlyA haemolysin of Escherichia coli $[1,21,84]$. This protein is secreted via an oligomeric complex composed of an inner membrane ATP-binding cassette (ABC) exporter (IM-ABC) called HlyB, a membrane fusion protein (MFP) and an outer membrane protein TolC. This oligomeric complex spans both the inner and outer membrane but requires substrate binding for the assembly of the three components.

The type II secretion system can be exemplified by the pullulanase enzyme, PulA, from Klebsiella oxytoca [15,57]. PulA secretion is a two-step process where the protein is first exported into the periplasmic space via the SecBdependent pathway and subsequently transported across the outer membrane by a dedicated secretion apparatus composed of 12-16 proteins called the secreton. Recently, it has been demonstrated that, contrary to the original model, the Tat pathway could be used, in addition to the SecB-dependent pathway, for protein translocation across the inner membrane. The type II secretion system is also termed the secreton-dependent pathway (SDP) [82], and often incorrectly termed the general secretory pathway [75].

The type III secretion system (TTSS) is composed of a complex protein structure spanning both the inner and the outer membranes [7,50]. One of the main features of this transport system is that it can allow the direct injection of protein into a host eukaryotic cell. By acting as a molecular syringe this apparatus has also been called the injectisome. It should be pointed out this system is also involved in (i) the secretion of proteins into the extracellular milieu, (ii) flagella biogenesis, and (iii) Hpr pilus biogenesis.
The type IV secretion system (TFSS) is related to the systems involved in conjugative transfer of DNA [85]. It allows the secretion of (i) nucleoprotein DNA conjugation intermediates, (ii) multi-subunit toxins such as the pertussis toxin (PT) or (iii) monomeric protein such as RecA. Recently, this pathway has been divided into two subclasses: (i) type $\mathrm{IV}_{\mathrm{a}}$ corresponds to machinery assembly containing VirB homologues of Agrobacterium tumefaciens, and (ii) type $\mathrm{IV}_{\mathrm{b}}$ corresponds to functional secretion systems assembled from Tra homologues of the Incl ColIb-P9 plasmid of Shigella flexneri [10]. Proteins using this pathway can be secreted either into the extracellular milieu or directly into a host cell.

The type V secretion system represents the largest family of protein-translocating outer membrane porins in Gramnegative bacteria and the simplest secretion apparatus [86]. Proteins using this system are translocated across the outer membrane via a transmembrane pore formed by a selfencoded $\beta$-barrel structure.

\section{Autotransporter protein secretion}

This secretion process was first described for the IgA1 protease produced by Neisseria gonorrhoeae [56]. In the course of secretion, the $\operatorname{IgA} 1$ protease is modified at both its N-terminus and C-terminus. An N-terminal 27 aminoacid signal sequence is cleaved during the exportation of the protein by the Sec system while, in the extracellular milieu, the $\mathrm{C}$-terminus domain of the mature protein is missing. It was proposed that the C-terminus domain serves as a pore for the secretion of the mature protein through the outer membrane. The $\operatorname{IgA} 1$ protease is released into the extracellular milieu by an autocatalytic activity. Since no energy coupling or accessory factor seemed to be required for the translocation process, proteins secreted in this way received the name of autotransporters.

Since this seminal work, a large number of proteins have been identified as autotransporters. Indeed, autotransporters represent the most important group of secreted proteins in Gram-negative bacteria [86]. However, from a phylogenetic point of view this group is restricted to the phylum Proteobacteria, including the $\alpha-, \beta-, \gamma$ - and $\varepsilon$-proteobacteria classes, and to the phylum Chlamydiae [26]. Nevertheless, in all cases the primary structure of autotransporters is modular and composed of three domains [29] (Fig. 1A): the signal sequence, the passenger domain and the translocation unit. At the N-terminus, the signal sequence allows targeting of the protein to the inner membrane for its export into the periplasmic space. The passenger domain confers the diverse effector functions to the autotransporters. The translocation unit is the last main domain located at the C-terminal end of the protein. It consists of a short linker region having an $\alpha-$ helical secondary structure and a $\beta$-domain that will adopt a $\beta$-barrel secondary structure when embedded in the outer membrane $[49,54]$. The autotransporters are synthesised in a pre-pro-protein form in the bacterial cytoplasm and released 
EM

OM

Peri

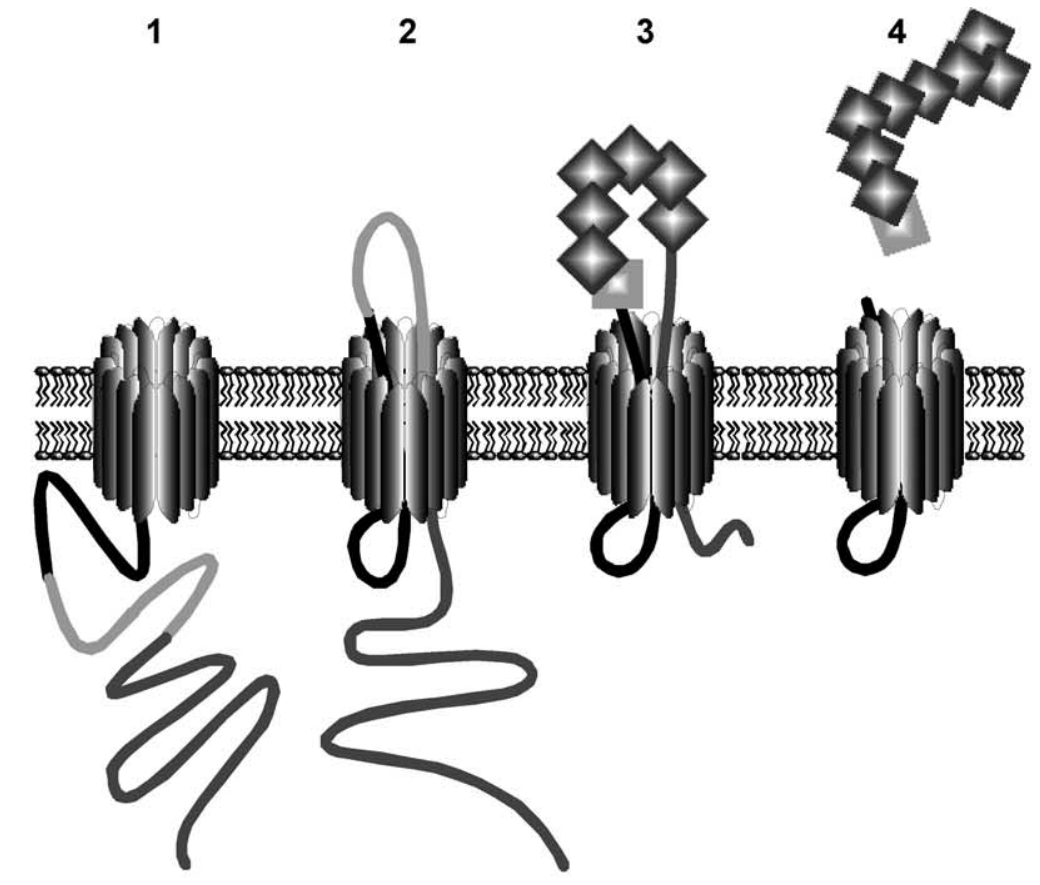

A

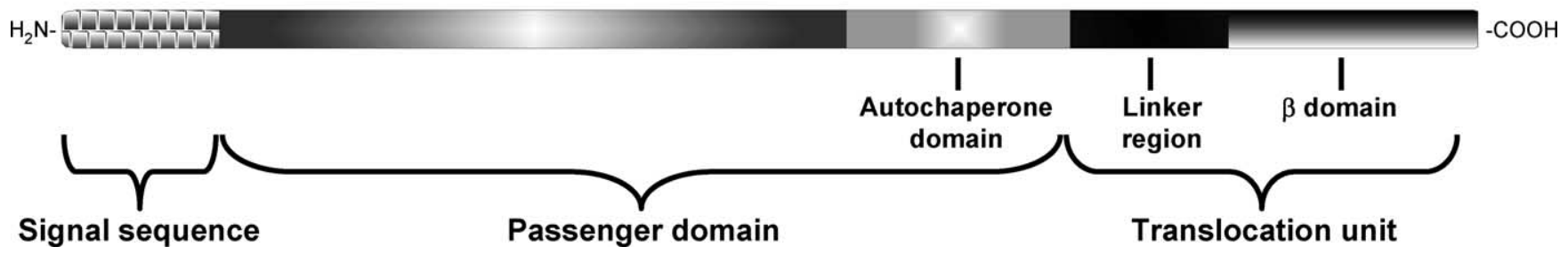

Fig. 1. The autotransporter secretion pathway. (A) The primary structure of an autotransporter. Each of the main domains involved in secretion is indicated. (B) Passenger domain secretion across the outer membrane. Step 1: the insertion of the $\beta$-domain into the outer membrane and formation of the $\beta$-barrel pore. Step 2: the linker region leads the secretion through the pore. Step 3: the autochaperone domain triggers the passenger domain folding as it emerges from the $\beta$-barrel. Step 4: the folded passenger domain is exposed on the cell surface and the effector molecule is released into the extracellular milieu after cleavage by a protease activity. Peri: periplasm; OM: outer membrane; EM: extracellular milieu. The square symbolises the folded state of the polypeptide.

in the periplasm in a pro-protein form. The translocation through the outer membrane completes the secretion of the autotransporters from the cytoplasm to the extracellular milieu. Depending on the autotransporter, the pro-protein may then be cleaved from the $\beta$-barrel domain or remain associated with the domain.

\subsection{Export into the periplasm}

Bioinformatic analyses of the extreme N-terminal sequence of most autotransporter proteins revealed that this region exhibits features of a prototypical signal sequence of the type associated with SecB-mediated translocation through the inner membrane [29]. From their N-terminus, these signal sequences typically possess: (i) an n-domain with positively charged residues, (ii) a hydrophobic core domain called h-domain, and (iii) a c-domain with a consensus signal peptidase recognition site [18]. However, some autotransporters exhibit unusually long signal sequences consisting of about 50 amino acids, as opposed to the 18-
26 residues generally encountered for the prototypical signal sequences. All of these atypical signal sequences, consisting of at least 42 amino acids [54], possess a conserved extension of their n-domain beginning with a MNKIYS$\mathrm{LKY}(\mathrm{S} / \mathrm{C} / \mathrm{H})$ motif, followed closely by a second motif containing conserved aromatic and hydrophobic residues, i.e., GLIAVSELAR [29]. It has long been suggested that these unusual features may impose additional properties such as secretion through the inner membrane in a Secindependent manner [19] or recruiting of other accessory proteins like SRP [29].

Investigating the role of the extended signal sequence of the haemoglobin protease autotransporter ( $\mathrm{Hbp}$ ) from $E$. coli, Sijbrandi et al. demonstrated that the targeting and translocation mechanism through the inner membrane involved the targeting factor SRP and the Sec translocon [69]. $\mathrm{SecB}$ was not required for the targeting of $\mathrm{Hbp}$, but it could compensate to some extent for the absence of a functional SRP pathway. The co-translational translocation of the SRP pathway might prevent degradation or premature folding of 
Hbp in the cytoplasm. This study presented the first example of an extracellular protein being targeted by SRP, which was previously only thought to be involved in inner membrane protein integration [14].

\subsection{Outer membrane translocation}

Based on the mechanism of porin biogenesis [74], it has been suggested that, following its export through the inner membrane, the autotransporter pro-protein interacts with the hydrophobic environment of the outer membrane, promoting its spontaneous insertion and folding in a thermodynamically favoured $\beta$-barrel conformation into the outer membrane [29]. The original model predicts that the first of the $\beta$-stands passes from the periplasmic space to the outer membrane thus leaving the passenger domain temporarily extending into the periplasm. At this point, it should be stressed that the issues of the folding state of the passenger domain, the formation and topology of the $\beta$-barrel as well as the secretion of the passenger domain are intimately linked.

Recently, the first description of an assisted folding pathway for integral outer membrane proteins (OMP) has been proposed [6]. In this model, the periplasmic chaperone Skp binds to the OMP, maintaining it in a soluble unfolded state before interacting with lipopolysaccharide (LPS) molecules to form a folding-competent intermediate that inserts and folds in the lipid bilayer of the outer membrane. However, this model requires modification due to the recent discovery of the involvement of the surface antigen Omp85 in OMP assembly [83]. Omp85 appears essential for general OMP assembly, including autotransporters, which challenges the concept of spontaneous insertion of OMP. However, the molecular mechanisms involved behind this function remain to be elucidated.

During the insertion of the $\beta$-domain into the lipid bilayer of the outer membrane, the first and last $\beta$-strands form hydrogen bonds in an antiparallel fashion to close the ring conformation, permitting the establishment of a molecular pore [29]. The alternating hydrophobic side chains of amino acids are embedded within the hydrophobic lipid bilayer while the hydrophilic side chains project into an aqueous environment in the centre of the $\beta$-barrel. The consensus motif $(\mathrm{Y} / \mathrm{V} / \mathrm{I} / \mathrm{F} / \mathrm{W}) \mathrm{X}(\mathrm{F} / \mathrm{W})$ found at the extreme carboxyl terminus, also found in other OMPs, appears very important for protein folding and stabilization of the monomer permitting the proper outer membrane localization of the protein $[13,30,71]$. The $\beta$-domain of autotransporters generally consists of 250-300 amino-acid residues. From bioinformatic analyses it has been predicted that the $\beta$-barrel of most autotransporters exhibit 14 antiparallel amphipathic strands consisting of 9 to 12 residues $[47,86]$. Still, the exact shape and conformation of the autotransporter $\beta$-barrel is subject to speculation but hopefully this should be resolved in the near future through current efforts which are being made by several research teams to crystallise this domain.
In the original model outer membrane translocation was proposed to be a two-step process where insertion of the C-terminus translocation unit occurs in the outer membrane and then translocation of the unfolded passenger domain occurs through the hydrophilic channel formed by the monomeric $\beta$-barrel [39-42]. Translocation of the protein through the pore formed by the $\beta$-domain was assumed to be incompatible with the native conformation of the passenger domain. However, by using a single-chain antibody as a reporter passenger domain instead of the IgA1 protease functional domain, it was concluded that the folding of the passenger domain takes place in the periplasm before, or at least simultaneously with, translocation through the outer membrane [80]. It also appeared unlikely that the folding of the passenger domain in the extracellular environment would provide the energy required for the translocation through the outer membrane. Reinvestigating the secretion of IgA1 protease, it was recently proposed that the passenger domain was instead secreted through an oligomeric ring-shaped structure consisting of a minimum of $6 \beta$-barrels and forming a central hydrophilic $\sim 2 \mathrm{~nm}$ diameter pore [81]. The exact structure of the oligomeric ring remains speculative since it has also been proposed that it could consist of $8-10$ monomers. The pore was proposed to be large enough to tolerate the passage of proteins in a folded state. However, the IgA1 protease is the only autotransporter for which a quaternary structural organisation of the $\beta$-barrels has been suggested.

From the study of the autotransporter PrtS from Serratia marcescens, however, it was demonstrated that the folding of the passenger domain only occurs on the bacterial cell surface and implied a putative intramolecular chaperone region [52]. A recent investigation by Oliver et al. of the BrkA autotransporter from Bordetella pertussis has definitively revealed the presence of an intramolecular chaperone, the PD002475 domain, in several autotransporters, including the IgA1 protease [55]. This domain is essential for the folding of the passenger domain, probably by acting as an intramolecular building block triggering or initiating correct folding of the passenger domain. Results of this investigation also suggest that previous findings by Veiga et al. [80] were probably biased by the study of a passenger domain unrelated to the autotransporters. From this study, a model of translocation has been proposed where the passenger domain remains unfolded, or partially folded, as it transits through the channel formed by the monomeric $\beta$-barrel and the folding, triggered by the autochaperone domain, begins vectorally in a $\mathrm{C}$-terminal direction as the passenger domain emerges from the pore on the bacterial surface (Fig. 1B). This also raises the possibility that a common sorting mechanism assisting the assembly and export of autotransporter proteins could involve the autochaperone domain.

In addition to the $\beta$-barrel, which permits the translocation of the passenger domain, the linker region found upstream of the $\beta$-domain is essential to secretion. Together these two domains form the translocation unit [49,54]. 
Rather than the $\beta$-domain, the translocation unit is a more appropriate operational definition for the transporter domain. The linker region consists of a 21 to 39 amino acids $\alpha$-helical region preceding the $\beta$-domain, and is probably involved in the formation of a hair-pin structure that leads secretion of the passenger domain through the channel formed by the $\beta$ barrel [29].

\subsection{Maturation on the cell surface}

Once at the bacterial surface, cleavage between the passenger domain and the translocation unit can occur either well upstream or within the linker region [54]. Once cleaved the passenger is either released into the extracellular medium or can remain in contact with the bacterial cell surface. In the case of BrkA the passenger domain remains steadfastly associated with the bacterial outer membrane, probably via the linker region which could act as an anchor. For some autotransporters, like AIDA-I and Antigen 43 from E. coli, the passenger domain remains in contact with the bacterial surface via noncovalent interactions with the $\beta$-domain. The passenger domain is not necessarily cleaved but can also remain intact as a large protein with a membrane-bound $\mathrm{C}$-terminal domain and an $\mathrm{N}$-terminal domain extending into the extracellular milieu, like the autotransporter Hia from Haemophilus influenzae [70]. Cleavage of the passenger domain is the result of either autocatalytic activity [66] or a membrane-bound protease [67]. These two systems are apparently not exclusive of one another since for SepA, an autotransporter from Shigella flexneri, the proteases could cleave the passenger domain in the absence of the autocatalytic activity [3]. As observed with the autotransporter Hap from Haemophilus influenzae, the passenger domain can be processed at several sites and possibly by a variety of protease [30].

Once the passenger domain is released, it can mature further. To achieve an active state, the passenger domain of IgA1 protease is cleaved into the functional $106-\mathrm{kDa}$ protease and a small stable $\alpha$-protein [56]. A similar example of further maturation applies to VacA, the vacuolating cytotoxin of Helicobacter pylori. In this case the ca. $90-\mathrm{kDa}$ passenger domain is further divided into two separable subdomains, namely an amino terminal (proximal) $33 \mathrm{kDa}$ (p33) and a carboxy terminal (distal) $55 \mathrm{kDa}$ (p55) which oligomerises into a large functional complex [48]. Low $\mathrm{pH}$ appears to be important for the biological functionality of this toxin, in fact, under acidic conditions the dissociation and rearrangement of the VacA oligomeric complex results in enhanced internalization and cytotoxic activity [51,72].

The question of the fate of the $\beta$-barrel has not been addressed definitively in the literature. By analogy with other outer membrane proteins, the $\beta$-barrel of autotransporters might be a rather stable structure [65] and it can be speculated that, overtime, the accumulation of pore-forming structure in the outer membrane should be lethal for the bacteria and that some kind of degradation or regulation process should occur. Results from cellular localization seem to corroborate the persistence of the $\beta$-barrel in the outer membrane [9]. Since the N-terminal rule cannot apply [79], to date the degradation mechanism(s) of periplasmic and outer membrane proteins remains elusive and further investigation is required [46].

\section{Systems related to the autotransporter secretion pathway}

The type $\mathrm{V}$ secretion system family of proteins contains secreted proteins that (i) contain all the information for translocation through cell envelope and/or require single accessory factors, and (ii) are translocated across the outer membrane via a transmembrane pore formed by a $\beta$-barrel $[29,75]$. The proteins secreted by this pathway contain: (i) an $\mathrm{N}$-terminal signal sequence for inner membrane translocation, (ii) a functional passenger domain that can be surfaceexposed or released into the extracellular milieu, (iii) a linker region necessary for translocation of the passenger domain through the outer membrane, and (iv) a C-terminal region involved in the formation of a transmembrane pore. Based on these and others criteria, the two-partner system (TPS) and the Oca (oligomeric coiled coils adhesin) family have been grouped under the type $\mathrm{V}$ secretion umbrella as distinct subgroups [16,24,25,34,61].

\subsection{The two-partner system (TPS)}

In contrast to the autotransporter pathway where the protein is produced as a single polypeptide, in the TPS the passenger domain (also called the exoprotein) and the pore forming $\beta$-domain (also called transporter domain) are translated as two separate proteins, respectively referred as members of TpsA and TpsB families [37]. In the TPS, the exoprotein is translocated through the outer membrane via a $\beta$-barrel pore formed by TpsB [37]. The $\beta$-barrel topology appears different from the autotransporter $\beta$-domain as indicated by the prediction of 19 amphipathic $\beta$-strands in TpsB [23] and different conductance values [36]. TpsB possesses additional levels of complexity as suggested by its involvement in the maturation of the passenger domain into its active form [37] or by the presence of C-terminal extensions [17]. Beyond the signal peptide, the TPS implies specific recognition events between the exoprotein and the transporter domain that could permit further translocation through the outer membrane [20,35,64]. It is suggested that the exoprotein transits the periplasm in an unfolded conformation and folds progressively at the cell surface as it is translocated through the transporter domain [22]. Thus, the translocation across both membranes seems coupled and the free energy of folding might be the driving force for the outer membrane translocation [37]. The efficient extracellular release of the mature filamentous haemagglutinin (FHA) of Bordetella pertussis depends on the presence of a C-terminal 
domain which is proteolytically removed in the course of secretion. As observed in some autotransporters, this domain acts as an intramolecular chaperone to assist secretion by preventing premature folding [59]. In contrast, activation of the Serratia haemolysin ShlA does not require proteolytic processing but rather requires the addition of molecules of phophatidylethanolamine mediated by ShlB [31]. The TPS seems the most widespread of the outer membrane proteintranslocation porins since it has been identified in animal, plant and fungal kingdoms of eukaryotes as well as in several bacterial genera [86]. However, from an evolutionary point of view it is still difficult to discriminate between divergent evolution from a common ancestor of the autotransporters and the TPS or a convergent evolution towards a similar solution for secretion of large proteins across the outer membrane [12].

\subsection{The Oca family}

Utilising YadA as a prototype, the members of the Oca family have been recently described as a subfamily of surface-attached oligomeric autotransporters [61]. YadA possesses six different domains: (i) an N-terminal signal sequence, (ii) head-D, (iii) neck-D, (iv) stalk-D, (v) linking- $\mathrm{R}$ and, (vi) a C-terminal region consisting of only four $\beta$-strands. Deletion of the $\mathrm{C}$-terminal domain abolished membrane insertion of YadA [73] while deletion of the linker region results in the degradation of the whole protein [61]. This region forms a $\beta$-barrel pore consisting of $12 \beta$-strands after trimerisation and confers an overall lollipop-like structure on the proteins displayed on the cell surface [32]. This conformation corresponds to an additional topology which could be proposed for the type $\mathrm{V}$ secretion system.

\section{Concluding remarks}

The passenger domain of an autotransporter protein corresponds to the secreted effector molecule. To date, all of the functionally characterised autotransporters have been implicated in bacterial virulence either by (i) displaying enzymatic activity (protease, peptidase, lipase, esterase), (ii) mediating actin-promoted bacterial motility, or acting as (iii) adhesins, (iv) toxins/cytotoxins, (v) immunomodulatory proteins, or as more recently discovered, (vi) permitting maturation of another virulent protein $[11,27,29]$. The exoproteins of the TPS have been reported to be (i) adhesin, (ii) haemopexin:haem-binding proteins (iii) antigenic factors or (iv) haemolysins/cytolysins [37]. Proteins of the Oca family are essentially adhesins [61], but further investigations may reveal a wider family and greater diversity of functions.

Besides their virulence functions, several investigations have also highlighted the potential biotechnological exploitation of the autotransporter secretion pathway, namely by displaying heterologous proteins on the bacterial surface [68]. This autodisplay system has been used successfully for (i) the display of receptor or ligand for purification or binding assays [78], (ii) expression of peptide libraries for epitope mapping or antibody specificity test [44], (iii) functional domain analyses of heterologous proteins [8], (iv) bioconversion by expressing enzymatic activity on the bacterial surface [38], and (v) exposure of antigenic determinants for vaccine development [45]. Undoubtedly, both the secretion pathways, and the potential for these systems in producing heterologous proteins, need further investigation.

\section{Acknowledgements}

This work was supported in part by the Biotechnology and Biological Sciences Research Council (BBSRC) grants 81/D14955 and 81/P14130 to IH.

\section{References}

[1] C. Andersen, Channel-tunnels: Outer membrane components of type I secretion systems and multidrug efflux pumps of Gram-negative bacteria, Rev. Physiol. Biochem. Pharmacol. 147 (2003) 122-165.

[2] D. Beha, S. Deitermann, M. Muller, H.G. Koch, Export of betalactamase is independent of the signal recognition particle, J. Biol. Chem. 278 (2003) 22161-22167.

[3] Z. Benjelloun-Touimi, P.J. Sansonetti, C. Parsot, SepA, the major extracellular protein of Shigella flexneri: Autonomous secretion and involvement in tissue invasion, Mol. Microbiol. 17 (1995) 123-135.

[4] B.C. Berks, F. Sargent, T. Palmer, The Tat protein export pathway, Mol. Microbiol. 35 (2000) 260-274.

[5] T. Bruser, C. Sanders, An alternative model of the twin-arginine translocation system, Microbiol. Res. 158 (2003) 7-17.

[6] P.V. Bulieris, S. Behrens, O. Holst, J.H. Kleinschmidt, Folding and insertion of the outer membrane protein OmpA is assisted by the chaperone Skp and by lipopolysaccharide, J. Biol. Chem. 278 (2003) 9092-9099.

[7] D. Buttner, U. Bonas, Port of entry-the type III secretion translocon, Trends Microbiol. 10 (2002) 186-192.

[8] N. Casali, M. Konieczny, M.A. Schmidt, L.W. Riley, Invasion activity of a Mycobacterium tuberculosis peptide presented by the Escherichia coli AIDA autotransporter, Infect. Immun. 70 (2002) 6846-6852.

[9] I. Charles, N. Fairweather, D. Pickard, J. Beesley, R. Anderson, G. Dougan, M. Roberts, Expression of the Bordetella pertussis P69 pertactin adhesin in Escherichia coli: Fate of the carboxy-terminal domain, Microbiology 140 (1994) 3301-3308.

[10] P.J. Christie, J.P. Vogel, Bacterial type IV secretion: Conjugation systems adapted to deliver effector molecules to host cells, Trends Microbiol. 8 (2000) 354-360.

[11] L. Coutte, R. Antoine, H. Drobecq, C. Locht, F. Jacob-Dubuisson, Subtilisin-like autotransporter serves as maturation protease in a bacterial secretion pathway, EMBO J. 20 (2001) 5040-5048.

[12] A. Danchin, Genomes and evolution, Curr. Issues Mol. Biol. 5 (2003) 37-42.

[13] H. de Cock, M. Struyve, M. Kleerebezem, T. van der Krift, J. Tommassen, Role of the carboxy-terminal phenylalanine in the biogenesis of outer membrane protein PhoE of Escherichia coli K-12, J. Mol. Biol. 269 (1997) 473-478.

[14] J.W. de Gier, J. Luirink, Biogenesis of inner membrane proteins in Escherichia coli, Mol. Microbiol. 40 (2001) 314-322. 
[15] C. d'Enfert, C. Chapon, A.P. Pugsley, Export and secretion of the lipoprotein pullulanase by Klebsiella pneumoniae, Mol. Microbiol. 1 (1987) 107-116.

[16] M. Desvaux, N.J. Parham, I.R. Henderson, Le système de sécrétion de type V chez les bactéries Gram-, Biofutur (2003), in press.

[17] M. Domenighini, D. Relman, C. Capiau, S. Falkow, A. Prugnola, V. Scarlato, R. Rappuoli, Genetic characterization of Bordetella pertussis filamentous haemagglutinin: A protein processed from an unusually large precursor, Mol. Microbiol. 4 (1990) 787-800.

[18] P. Fekkes, A.J. Driessen, Protein targeting to the bacterial cytoplasmic membrane, Microbiol. Mol. Biol. Rev. 63 (1999) 161-173.

[19] R.C. Fernandez, A.A. Weiss, Cloning and sequencing of a Bordetella pertussis serum resistance locus, Infect. Immun. 62 (1994) 47274738.

[20] S. Grass, J.W. St. Geme 3rd, Maturation and secretion of the non-typable Haemophilus influenzae HMW1 adhesin: Roles of the N-terminal and C-terminal domains, Mol. Microbiol. 36 (2000) 5567.

[21] L. Gray, K. Baker, B. Kenny, N. Mackman, R. Haigh, I.B. Holland, A novel C-terminal signal sequence targets Escherichia coli haemolysin directly to the medium, J. Cell. Sci. 11 (Suppl.) (1989) 45-57.

[22] S. Guedin, E. Willery, C. Locht, F. Jacob-Dubuisson, Evidence that a globular conformation is not compatible with FhaC-mediated secretion of the Bordetella pertussis filamentous haemagglutinin, Mol. Microbiol. 29 (1998) 763-774.

[23] S. Guedin, E. Willery, J. Tommassen, E. Fort, H. Drobecq, C. Locht, F. Jacob-Dubuisson, Novel topological features of FhaC, the outer membrane transporter involved in the secretion of the Bordetella pertussis filamentous hemagglutinin, J. Biol. Chem. 275 (2000) 30202-30210.

[24] I.R. Henderson, R. Cappello, J.P. Nataro, Autotransporter proteins, evolution and redefining protein secretion, Trends Microbiol. 8 (2000) $529-532$.

[25] I.R. Henderson, R. Cappello, J.P. Nataro, Autotransporter proteins, evolution and redefining protein secretion: Response, Trends Microbiol. 8 (2000) 534-535.

[26] I.R. Henderson, A.C. Lam, Polymorphic proteins of Chlamydia spp.- - autotransporters beyond the Proteobacteria, Trends Microbiol. 9 (2001) 573-578.

[27] I.R. Henderson, J.P. Nataro, Virulence functions of autotransporter proteins, Infect. Immun. 69 (2001) 1231-1243

[28] I.R. Henderson, J.P. Nataro, J.B. Kaper, T.F. Meyer, S.K. Farrand, D.L. Burns, B.B. Finlay, J.W. St. Geme 3rd, Renaming protein secretion in the Gram-negative bacteria, Trends Microbiol. 8 (2000) 352.

[29] I.R. Henderson, F. Navarro-Garcia, J.P. Nataro, The great escape: Structure and function of the autotransporter proteins, Trends Microbiol. 6 (1998) 370-378.

[30] D.R. Hendrixson, M.L. de la Morena, C. Stathopoulos, J.W. St. Geme 3rd, Structural determinants of processing and secretion of the Haemophilus influenzae hap protein, Mol. Microbiol. 26 (1997) 505-518.

[31] R. Hertle, S. Brutsche, W. Groeger, S. Hobbie, W. Koch, U. Konninger, V. Braun, Specific phosphatidylethanolamine dependence of Serratia marcescens cytotoxin activity, Mol. Microbiol. 26 (1997) 853-865.

[32] E. Hoiczyk, A. Roggenkamp, M. Reichenbecher, A. Lupas, J. Heesemann, Structure and sequence analysis of Yersinia YadA and Moraxella UspAs reveal a novel class of adhesins, EMBO J. 19 (2000) 5989-5999.

[33] K. Ito, M. Wittekind, M. Nomura, K. Shiba, T. Yura, A. Miura, H. Nashimoto, A temperature-sensitive mutant of E. coli exhibiting slow processing of exported proteins, Cell 32 (1983) 789-797.

[34] F. Jacob-Dubuisson, R. Antoine, C. Locht, Autotransporter proteins, evolution and redefining protein secretion: Response, Trends Microbiol. 8 (2000) 533-534.

[35] F. Jacob-Dubuisson, C. Buisine, E. Willery, G. Renauld-Mongenie, C. Locht, Lack of functional complementation between Bordetella pertussis filamentous hemagglutinin and Proteus mirabilis HpmA hemolysin secretion machineries, J. Bacteriol. 179 (1997) 775-783.
[36] F. Jacob-Dubuisson, C. El-Hamel, N. Saint, S. Guedin, E. Willery, G. Molle, C. Locht, Channel formation by FhaC, the outer membrane protein involved in the secretion of the Bordetella pertussis filamentous hemagglutinin, J. Biol. Chem. 274 (1999) 37731-37735.

[37] F. Jacob-Dubuisson, C. Locht, R. Antoine, Two-partner secretion in Gram-negative bacteria: A thrifty, specific pathway for large virulence proteins, Mol. Microbiol. 40 (2001) 306-313.

[38] J. Jose, R. Bernhardt, F. Hannemann, Cellular surface display of dimeric Adx and whole cell P450-mediated steroid synthesis on E. coli, J. Biotechnol. 95 (2002) 257-268.

[39] T. Klauser, J. Kramer, K. Otzelberger, J. Pohlner, T.F. Meyer, Characterization of the Neisseria IgA beta-core: The essential unit for outer membrane targeting and extracellular protein secretion, J. Mol. Biol. 234 (1993) 579-593.

[40] T. Klauser, J. Pohlner, T.F. Meyer, Extracellular transport of cholera toxin B subunit using Neisseria IgA protease beta-domain: Conformation-dependent outer membrane translocation, EMBO J. 9 (1990) 1991-1999.

[41] T. Klauser, J. Pohlner, T.F. Meyer, The secretion pathway of $\operatorname{IgA}$ protease-type proteins in Gram-negative bacteria, Bioessays 15 (1993) 799-805.

[42] T. Klauser, J. Pohlner, T.F. Meyer, Selective extracellular release of cholera toxin B subunit by Escherichia coli: Dissection of Neisseria IgA beta-mediated outer membrane transport, EMBO J. 11 (1992) 2327-2335.

[43] H.G. Koch, M. Moser, M. Muller, Signal recognition particledependent protein targeting, universal to all kingdoms of life, Rev. Physiol. Biochem. Pharmacol. 146 (2003) 55-94.

[44] M.P. Konieczny, M. Suhr, A. Noll, I.B. Autenrieth, M.A. Schmidt, Cell surface presentation of recombinant (poly-)peptides including functional T-cell epitopes by the AIDA autotransporter system, FEMS Immunol. Med. Microbiol. 27 (2000) 321-332.

[45] U. Kramer, K. Rizos, H. Apfel, I.B. Autenrieth, C.T. Lattemann, Autodisplay: Development of an efficacious system for surface display of antigenic determinants in Salmonella vaccine strains, Infect. Immun. 71 (2003) 1944-1952.

[46] A.J. Link, K. Robison, G.M. Church, Comparing the predicted and observed properties of proteins encoded in the genome of Escherichia coli $\mathrm{K}-12$, Electrophoresis 18 (1997) 1259-1313.

[47] B.J. Loveless, M.H. Saier Jr., A novel family of channel-forming, autotransporting, bacterial virulence factors, Mol. Membr. Biol. 14 (1997) 113-123.

[48] P. Lupetti, J.E. Heuser, R. Manetti, P. Massari, S. Lanzavecchia, P.L. Bellon, R. Dallai, R. Rappuoli, J.L. Telford, Oligomeric and subunit structure of the Helicobacter pylori vacuolating cytotoxin, J. Cell. Biol. 133 (1996) 801-807.

[49] J. Maurer, J. Jose, T.F. Meyer, Characterization of the essential transport function of the AIDA-I autotransporter and evidence supporting structural predictions, J. Bacteriol. 181 (1999) 7014-7020.

[50] T. Michiels, P. Wattiau, R. Brasseur, J.M. Ruysschaert, G. Cornelis, Secretion of Yop proteins by Yersiniae, Infect. Immun. 58 (1990) 2840-2849.

[51] M. Molinari, C. Galli, M. de Bernard, N. Norais, J.M. Ruysschaert, R. Rappuoli, C. Montecucco, The acid activation of Helicobacter pylori toxin VacA: Structural and membrane binding studies, Biochem. Biophys. Res. Commun. 248 (1998) 334-340.

[52] Y. Ohnishi, M. Nishiyama, S. Horinouchi, T. Beppu, Involvement of the COOH-terminal pro-sequence of Serratia marcescens serine protease in the folding of the mature enzyme, J. Biol. Chem. 269 (1994) 32800-32806.

[53] D.B. Oliver, J. Beckwith, Identification of a new gene (secA) and gene product involved in the secretion of envelope proteins in Escherichia coli, J. Bacteriol. 150 (1982) 686-691.

[54] D.C. Oliver, G. Huang, R.C. Fernandez, Identification of secretion determinants of the Bordetella pertussis BrkA autotransporter, J. Bacteriol. 185 (2003) 489-495. 
[55] D.C. Oliver, G. Huang, E. Nodel, S. Pleasance, R.C. Fernandez, A conserved region within the Bordetella pertussis autotransporter BrkA is necessary for folding of its passenger domain, Mol. Microbiol. 47 (2003) 1367-1383.

[56] J. Pohlner, R. Halter, K. Beyreuther, T.F. Meyer, Gene structure and extracellular secretion of Neisseria gonorrhoeae IgA protease, Nature 325 (1987) 458-462.

[57] A.P. Pugsley, The complete general secretory pathway in Gramnegative bacteria, Microbiol. Rev. 57 (1993) 50-108.

[58] H.Y. Qi, J.B. Hyndman, H.D. Bernstein, DnaK promotes the selective export of outer membrane protein precursors in SecA-deficient Escherichia coli, J. Biol. Chem. 277 (2002) 51077-51083.

[59] G. Renauld-Mongenie, J. Cornette, N. Mielcarek, F.D. Menozzi, C. Locht, Distinct roles of the N-terminal and C-terminal precursor domains in the biogenesis of the Bordetella pertussis filamentous hemagglutinin, J. Bacteriol. 178 (1996) 1053-1060.

[60] C. Robinson, A. Bolhuis, Protein targeting by the twin-arginine translocation pathway, Nat. Rev. Mol. Cell. Biol. 2 (2001) 350-356.

[61] A. Roggenkamp, N. Ackermann, C.A. Jacobi, K. Truelzsch, H. Hoffmann, J. Heesemann, Molecular analysis of transport and oligomerization of the Yersinia enterocolitica adhesin YadA, J. Bacteriol. 185 (2003) 3735-3744.

[62] M.H. Saier Jr., A functional-phylogenetic classification system for transmembrane solute transporters, Microbiol. Mol. Biol. Rev. 64 (2000) 354-411.

[63] G.P. Salmond, P.J. Reeves, Membrane traffic wardens and protein secretion in Gram-negative bacteria, Trends Biochem. Sci. 18 (1993) $7-12$.

[64] R. Schonherr, R. Tsolis, T. Focareta, V. Braun, Amino acid replacements in the Serratia marcescens haemolysin ShIA define sites involved in activation and secretion, Mol. Microbiol. 9 (1993) 12291237.

[65] G.E. Schulz, The structure of bacterial outer membrane proteins, Biochim. Biophys. Acta 1565 (2002) 308-317.

[66] D. Serruto, J. Adu-Bobie, M. Scarselli, D. Veggi, M. Pizza, R. Rappuoli, B. Arico, Neisseria meningitidis App, a new adhesin with autocatalytic serine protease activity, Mol. Microbiol. 48 (2003) 323334.

[67] K.D. Shere, S. Sallustio, A. Manessis, T.G. D’Aversa, M.B. Goldberg, Disruption of IcsP, the major Shigella protease that cleaves IcsA, accelerates actin-based motility, Mol. Microbiol. 25 (1997) 451-462.

[68] K. Shimada, Y. Ohnishi, S. Horinouchi, T. Beppu, Extracellular transport of pseudoazurin of Alcaligenes faecalis in Escherichia coli using the COOH-terminal domain of Serratia marcescens serine protease, J. Biochem. (Tokyo) 116 (1994) 327-334.

[69] R. Sijbrandi, M.L. Urbanus, C.M. ten Hagen-Jongman, H.D. Bernstein, B. Oudega, B.R. Otto, J. Luirink, Signal recognition particle (SRP)-mediated targeting and Sec-dependent translocation of an extracellular Escherichia coli protein, J. Biol. Chem. 278 (2003) 46544659.

[70] J.W. St. Geme 3rd, D. Cutter, The Haemophilus influenzae Hia adhesin is an autotransporter protein that remains uncleaved at the $\mathrm{C}$ terminus and fully cell associated, J. Bacteriol. 182 (2000) 6005-6013.
[71] M. Struyve, M. Moons, J. Tommassen, Carboxy-terminal phenylalanine is essential for the correct assembly of a bacterial outer membrane protein, J. Mol. Biol. 218 (1991) 141-148.

[72] I. Szabo, S. Brutsche, F. Tombola, M. Moschioni, B. Satin, J.L. Telford, R. Rappuoli, C. Montecucco, E. Papini, M. Zoratti, Formation of anion-selective channels in the cell plasma membrane by the toxin VacA of Helicobacter pylori is required for its biological activity, EMBO J. 18 (1999) 5517-5527.

[73] A. Tamm, A.M. Tarkkanen, T.K. Korhonen, P. Kuusela, P. Toivanen, M. Skurnik, Hydrophobic domains affect the collagen-binding specificity and surface polymerization as well as the virulence potential of the YadA protein of Yersinia enterocolitica, Mol. Microbiol. 10 (1993) 995-1011.

[74] L.K. Tamm, A. Arora, J.H. Kleinschmidt, Structure and assembly of beta-barrel membrane proteins, J. Biol. Chem. 276 (2001) 3239932402.

[75] D.G. Thanassi, S.J. Hultgren, Multiple pathways allow protein secretion across the bacterial outer membrane, Curr. Opin. Cell. Biol. 12 (2000) 420-430.

[76] Q.A. Valent, Signal recognition particle mediated protein targeting in Escherichia coli, Antonie Van Leeuwenhoek 79 (2001) 17-31.

[77] Q.A. Valent, P.A. Scotti, S. High, J.W. de Gier, G. von Heijne, G. Lentzen, W. Wintermeyer, B. Oudega, J. Luirink, The Escherichia coli SRP and SecB targeting pathways converge at the translocon, EMBO J. 17 (1998) 2504-2512.

[78] M. Valls, S. Atrian, V. de Lorenzo, L.A. Fernandez, Engineering a mouse metallothionein on the cell surface of Ralstonia eutropha $\mathrm{CH} 34$ for immobilization of heavy metals in soil, Nat. Biotechnol. 18 (2000) $661-665$.

[79] A. Varshavsky, The N-end rule: Functions, mysteries, uses, Proc. Natl. Acad. Sci. USA 93 (1996) 12142-12149.

[80] E. Veiga, V. de Lorenzo, L.A. Fernandez, Probing secretion and translocation of a beta-autotransporter using a reporter single-chain $\mathrm{Fv}$ as a cognate passenger domain, Mol. Microbiol. 33 (1999) 1232-1243.

[81] E. Veiga, E. Sugawara, H. Nikaido, V. de Lorenzo, L.A. Fernandez, Export of autotransported proteins proceeds through an oligomeric ring shaped by C-terminal domains, EMBO J. 21 (2002) 2122-2131.

[82] R. Voulhoux, G. Ball, B. Ize, M.L. Vasil, A. Lazdunski, L.F. Wu, A. Filloux, Involvement of the twin-arginine translocation system in protein secretion via the type II pathway, EMBO J. 20 (2001) 67356741.

[83] R. Voulhoux, M.P. Bos, J. Geurtsen, M. Mols, J. Tommassen, Role of a highly conserved bacterial protein in outer membrane protein assembly, Science 299 (2003) 262-265.

[84] C. Wandersman, P. Delepelaire, TolC, an Escherichia coli outer membrane protein required for hemolysin secretion, Proc. Natl. Acad. Sci. USA 87 (1990) 4776-4780.

[85] J.E. Ward, D.E. Akiyoshi, D. Regier, A. Datta, M.P. Gordon, E.W. Nester, Characterization of the virB operon from an Agrobacterium tumefaciens Ti plasmid, J. Biol. Chem. 263 (1988) 5804-5814.

[86] M.R. Yen, C.R. Peabody, S.M. Partovi, Y. Zhai, Y.H. Tseng, M.H. Saier, Protein-translocating outer membrane porins of Gram-negative bacteria, Biochim. Biophys. Acta 1562 (2002) 6-31. 